\title{
Internet of things in marketing and retail
}

\author{
[Nataša Đurđević, Aleksandra Labus, Zorica Bogdanović, Marijana Despotović-Zrakić]
}

\begin{abstract}
Internet of Things (IoT) is predicted to be one of the main raising megatrends in technology. Combined with current and expected ubiquity and pervasiveness of connected smart devices it gives ground to application in many areas, including marketing. Potential of sensors and actuators for context relevant, personalized, real-time, interactive communication with consumers and shoppers is seen as a driver of traffic and transaction, facilitator of movement along the path to purchase, able to enhance their experience. At the same time, they can serve to improve logistics and infrastructure for more effective retail business and store management. This paper reviews and organizes potential applications of IoT technologies and solutions for effective marketing at retail from the angle of manufacturers and retailers as well as from the angle of consumer/shopper facing or infrastructure and process related initiatives. We propose a model that indicates the potential that IoT has vs standard industry practices of marketing and retail to drive business results and competitive advantage. Proposed model is applied on multiple industry examples. With theoretical framework and practical examples, the paper provides relevant theory and managerial implications of IoT in marketing and retail.
\end{abstract}

Keywords - Internet of Things, marketing, context-aware computing, mobile marketing, geo-targeting, personalization

\section{Introduction}

Internet of Things (IoT) is predicted to be one of the main raising megatrends in technology $[1,2]$. Combined with current and expected ubiquity and pervasiveness of connected smart devices it gives ground to application in many areas: smart cities, smart homes, health, transportation, industrial automation [2, 3, 4], as well as retail and marketing. Technological development has enabled IoT technologies decreasing costs, increasing devices' computing power, cloud services, interoperability and security and privacy improvement $[3,5,6,7]$. These factors contribute to decreasing technology, organizational and financial barriers to expand IoT in marketing and retail industries.

Main expectation from IoT benefits in marketing and retail is seen in the potential of sensors and actuators for context relevant, personalized, real-time, interactive communication with consumers and shoppers as a driver of traffic and transaction, facilitator of movement along the path to purchase, able to enhance their experience $[8,9]$. At the same time, IoT can serve to improve logistics and infrastructure for more effective retail business and store management $[8,19,25,27,28,41]$.

Natasa Đurđević

*Aleksandra Labus

Zorica Bogdanović

Marijana Despotović-Zrakić

Faculty of Organizational Sciences, University of Belgrade Serbia
The aim of this paper is to show the examples and to propose a model of IoT application in marketing and retail. Proposed model is based on relevant business practices and technology solutions, connecting the manufacturers and retailers activating potential shoppers throughout the purchase cycle, combining the user interface and internal business processes. The model is validated on an experimental activation project and its measurement system.

\section{Literature Review}

The Internet of Things (IoT) is a dynamic global information network consisting of Internet connected objects, such as radio frequency identifications, sensors, and actuators, as well as other instruments and smart appliances that are becoming an integral component of the Internet [3]. The IoT is an intelligent network which connects all things to the Internet for the purpose of exchanging information and communicating through the information sensing devices in accordance with agreed protocols. It achieves the goal of intelligent identifying, locating, tracking, monitoring, and managing things [5]. The ultimate objective of this connectivity is to provide value-added services to users [15, 16]. Forecasts on the amount of installed IoT devices by the year 2020 vary from 21 to 212 billion [3, 6], and as much as $45 \%$ of all internet traffic by 2022 will be related to IoT. Current levels of implementation and investment levels into IoT by CPG (consumer packaged goods) and retail industry is lower than other sectors [6].

One of the important concepts related to IoT applications for marketing is context. IoT can sense contextual inputs and use them to deliver relevant information and services to the user [3, 17, 18]. The sensors integrated into ubiquitous mobile phones and tables, which have become integral part of people's lives, become the source of contextual information like location tracked via GPS [9, 19, 20]. Another important source of contextual information are social networks - combined with other contextual inputs from a mobile device this becomes a source for predicting behavior, recommendations or personal support [3].

For the purpose of focusing the attention of this paper to marketing and retail, we can focus the marketing to the field of shopper marketing: planning and execution of all marketing activities that influence a shopper along and beyond the path to purchase [9]. Together with IoT, mobile phones play an important role in this process: from the initial shopping trigger, to the purchase, consumption, repurchase and recommendations stages [9]. With a mobile device at hand, a shopper can initiate or leave the purchase cycle at any point, can do search, check products and obtain information, find out about prices, find store location and working hours, do the actual purchase, and check recommendations. The IoT system should enable the seamless shopping process and user experience even when the sensors, or users, or both are on the move [3, 21]. Customer experience and customer satisfaction are some of key success factors of marketing efforts, and they should be 
reached at any stage of the purchase cycle: pre, during or post purchase [10, 22].

IoT can have various applications in marketing and retail. We will split them into two segments: those directly consumer/shopper facing and those more related to business infrastructure and processes. The first segment contains following examples of practices: geo-targeting [19], personalized offers [4, 9], customized purchase experience [10, 22, 23], cross selling and up selling [9], tailored pricing [9], direct payment [24], automatic replenishment [9], drawing shoppers to store [9], in store digital interactive screens [22, 24], gamification [25], product and offer cocreation $[9,24]$. The second segment of infrastructure and process related practices contains following examples: shopper in store movement and behavior monitoring $[10,11$, $22,26]$, intelligent store ambient [10] and store layout [4], loyalty program [27], dynamic pricing [9], demographic and behavioral targeting [8], inventory and stock management $[4,8,9,12,13,22,25]$, supply chain management $[8,13$, $23,25]$, collaborative supply chain [28], tracking assets and equipment $[8,25]$, payment process $[8,24,25]$, in-store staff management [29], seamless cross-channel experience [7, 8, 29], real-time processes and activities monitoring [4, 8], new business models and revenue streams [23].

There are a number of different technology solutions for IoT: RFID, NFC, BLE, Wi-Fi, Z-wave and others [3]. Common requirements for mass IoT are small size, low energy use, computing power, connectivity, interoperability, security $[2,3,5,18,30,31]$. Protocols like RFID and NFC have been used in retail practice for inventory tracking or payments. BLE - Bluetooth Low Energy is a protocol that is attracting attention of retailers and marketers in recent years. As it has become the standard in most of current smart phones it lands itself for real-time, contextual, personalized communication and activation at or close to point of purchase, identifying microlocation $[9,11]$. BLE is a modification to the standard Bluetooth protocol to allow short range, low bandwidth, low latency, very efficient communication [30]. They are used when sending small amounts of data periodically is needed [3, 30]. IoT systems contain Application, Network and Perception layer [3] and comprise a number of component modules: interaction with local IoT devices, local analysis and processing, interaction with remote IoT devices, application specific data analysis and processing, integration into business processes and enterprise, user interface [2].

By its nature, IoT generate enormous amount of data. In order for this data to generate useful information and create value to the user [7], they should be connected and enabled via cloud services and big data analytics, comply with security and privacy requirements $[2,3,15,21]$. In addition, within the enterprise there should exist adequate organization: the culture, structure, role sort and talent [23, $32,33]$. It is not only corporate entities who can generate added value and financial gains out of data - consumers and shopper could have that same opportunity selling their own data generated by the intelligent devices in their homes and lives, for the benefits of personalized and relevant added services and experience [34].

From the literature review we can conclude that most of the identified academic papers focus on certain aspects of the IoT and/or marketing. Majority are addressing technology related topics, some are discussing specific application solutions for retail, or discussing contemporary marketing practices driven by technology advancement. We see a gap in bringing all these aspects in one comprehensive framework that would enable academia as well as practitioners to tackle the IoT implementation in marketing and retail business.

\section{Model of IoT Application in Marketing and Retail}

Academic papers related to IoT so far have focused more on technology related topics or specific narrow fields of implementation in retail or marketing. We have identified the gap of having a theoretical model of IoT application in marketing and retail that would be based on relevant business practices and technology solutions, connecting connect the manufacturers and retailers activating potential shoppers, combining the user interface and internal business processes. The developed model of IoT application in marketing and retail is given in Fig 1. Horizontally, it spans across the business process: from planning through implementation to measurement. Vertically, we review the IoT solutions from two aspects: consumer and shopper facing vs infrastructure and process related.

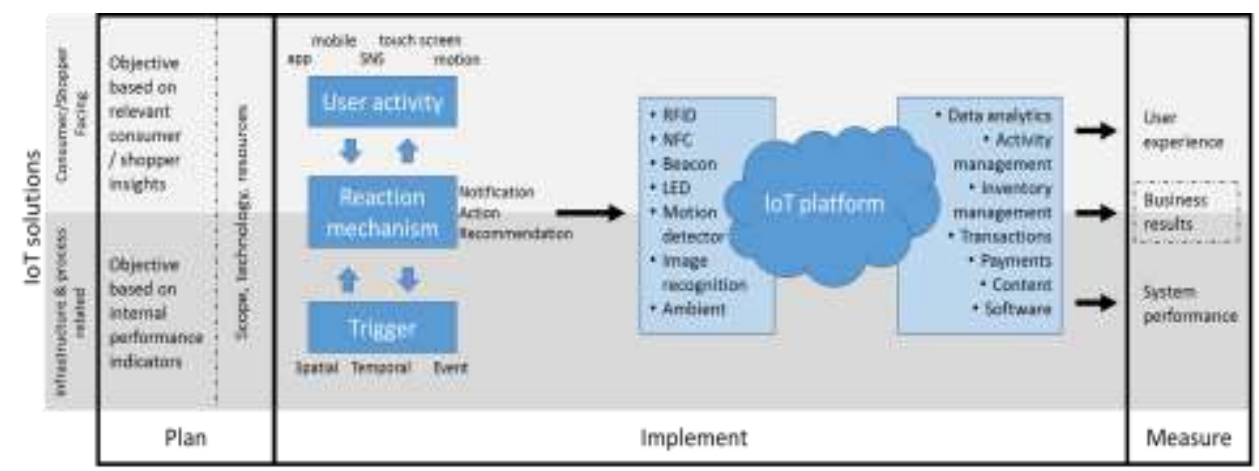

Figure 1. Model of IoT application in marketing and retail

Shown vertically, at plan stage, what needs to be defined is the clear objective of the IoT considered initiatives, having in mind the intended target: external for consumers and shopper, based on insights, or internal, targeting key performance indicators of corporate infrastructure and business process. Articulated objectives will indicate scope, technology solutions and resources needed - money, time and people. The focus of implementation stage is defining the reaction mechanism linking the sensor and the user and what should the IoT platform solve and provide. 
Based on [3], reaction mechanism can be notifications $(\mathrm{N})$, actions $(\mathrm{A})$ or recommendations $(\mathrm{R})$, while trigger can be spatial (S), temporal (T) or event based (E). Users/shoppers can be activated at any stage of the purchase cycle: before, during or post purchase [9]. Looking at the components [3], at perception layer are devices, network layer is the data exchange protocol and application layer is the service and analytics. The IoT platform assumes choice of actual IoT technology where each solution can have its benefits and limitations (RFID, or beacon, or motion detectors and other) and the business processes that have to be put in place to have the meaningful implementation and drive satisfactory business result. Massive data that will be generated with IoT have to be "tamed" with appropriate analytics and put to use for managerial interventions inventory, logistics, payments, content and so on.

Upon implementation, the enterprise should be able to assess the impact of IoT initiative and validate its contribution to long term business results, both in terms of direct financial result [33], as well as creating the sustainable competitive advantage. Main premise of successful marketing is creating the lasting recurring relationship with the customer, so understanding the IoT impact on our target consumers and shoppers is one area to measure, track and correct. Internally, system has to perform flawlessly and be supported by lasting organizational redesign. Finally, expectation is that IoT delivers incrementality to business performance measured by revenue and different efficiency and productivity ratios.

\section{Iv. Experiment Design For Model Validation}

In this section the theoretical framework of our model is applied on concrete business case. The case is related to a pilot project of shopper activation using beacons in partnership between the Coca-Cola Company (TCCC) and one of the leading retailers in Serbia.

In 2015 TCCC launched SimplyTastly mobile app in Serbia, developed by the Company as one of the relevant marketing tactics behind Coca-Cola and Food platform. It has been created as native app for Android and iOS because user experience and possibility to leverage device functionalities (location for geo-targeting, for example) were key objectives. Based on available extensive consumer and shopper research, application functionalities were designed to address shoppers' needs and they are: recipes search, shopping list creation, current retailers' leaflets and promotions review, store location, interactivity - questions, comments, content sharing, link with social networks profiles, given as illustration in Fig. 2. Our pilot project concept involves most of these functionalities.

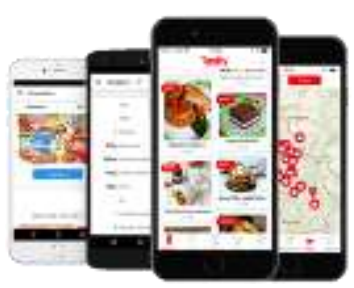

Figure 2. SimplyTastly functionalities
SimplyTastly has about 90 thousand loyal users in Serbia (app analytics show more than $80 \%$ of regular users), mainly in larger cities. Target audience for the app are women, mothers i.e. those who are responsible for household meals preparation and food supplies - they are also potential users of the Coca-Cola Company products and potential shoppers at selected retailer's supermarket and convenience outlets.

Details of the marketing program would be the following: with selected retail partner all outlets would have a special price offer for a bundle purchase of full meal items (for example, spaghetti + tomato sauce + minced meat + Coca-Cola, all together at discounted price), and this offer would have special secondary displays in stores and would be featured in the leaflet. Some of the outlets would have beacons placed. Users of SimplyTastly app would receive push notification about the offer. Based on in-app behavior analytics, all users searching for similar meals recipes or who are adding items from the offer to their shopping lists would receive notification about the offer and recommendation to add to basket and purchase. Objective of this phase would be to trigger the purchase, increase basket size with this bundle offer and generate traffic to retailer's stores. Further, when in vicinity of the beacon activated outlets, app users would be receiving notification on the offer triggered by the beacon in store. Accepting the notification, users would receive additional benefit of an extra discount digital coupon. Objective of this phase is for the IoT activation right before the purchase to trigger conversion. Meal combinations on offer would be rotated twice per week, and whole program would last for two months. Notifications would be activated only once in each promotional cycle.

The ultimate objective of using sensors for consumer activation is to generate incremental commercial results above and beyond standard promotional practices. To have such activations implemented at scale, they should be beneficial for all parties involved: manufacturer and retailer. Concrete features which are expected to generate incremental value are personalized offers based on in-app user behavior and utilizing new medium of communication - a sensor trigger just before the purchase. In addition to final commercial results, it is important to understand users' perceptions and attitudes. Assuming their in-app data sharing agreement, we should validate whether users see and value the benefits of personalized communication and offers, whether such communication is seen as acceptable or intrusive as well as whether there is an impact on attitudes towards brands included in the particular promotional program. Application of IoT is novelty for Serbian market. It is important to monitor and check functionality of all system components - sensors performance, exchange with users and servers, efficiency of the application algorithm and notifications, redemption of digital coupons.

To achieve above mentioned measurement targets, we would propose following methodology: in market test and volumetric modelling to track sales results, exit interviews with shoppers triggered by the beacon in store, and system performance tracking via existing database. Prior to market implementation, Coca-Cola Company employees would test the system within their offices for one week to validate the program criteria. Every marketing activity has its cost side. In addition to measuring separate elements of the project, 
full ROI (return on investment) should be performed to understand entire feasibility and scalability of the solution.

For the purpose of this paper, we will not develop the methodology details of each measurement areas - our goal is to review the different aspects of economical, psychological and technological effects in their totality.

\section{A. Measuring business result}

To be able to precisely measure the effect a beacon activation brings as a varying new factor in the promotional practice, we need to simultaneously track sales in stores with beacons and those without. All retail outlets would feature the same offer, but 10 outlets would be activated additionally with beacons. Since the promotion is planned to last for two months, 10 outlets tracked for 8 weeks gives enough observations to have appropriate quantification and enable sales modelling (based on previous references of similar models executed with Nielsen). For the purpose of objective measurement and understanding the causality of beacons to sales, digital coupons as well as in store execution quality (positions and management of display units), external agency partner should be engaged (Nielsen, for example). Defined success criteria would be statistically significant sales increase (measured as sell-out revenue) of all promoted categories and specifically soft drinks of the Coca-Cola Company.

Geographically the test would be implemented in Belgrade area for the following reasons: most SimplyTastly app users, logistical ease for beacons and displays placement and field monitoring, and largest number of retailer's outlets. Selected 10 stores should be of different format (supermarkets and convenience), should have standard turnover (based on retailer's historical data) and should be in frequent traffic areas. Upon selection of beacon activated stores, another 10 outlets representing their statistical "twins" by the parameters of location, turnover, format, equipment and implementation should be selected - this would be the reference control group to benchmark beacon activated outlets results against.

Analyzing digital coupons redemption (based on direct data from the retailer) vs activated phones i.e. users we will get the redemption rate, which is an important metric of marketing promotions success. The hypothesis is that this redemption rate will be higher than standard promotions redemption rate $(3-8 \%$ based on historical data).

\section{B. Measuring user experience}

Privacy, user control and transparency are critical for IoT acceptance in many areas in society [3, 15, 20, 31, 35]. For SimplyTastly app users receive transparent data policy and can choose to accept it when installing the app (the app does not require identification and revealing personal data). Initial trials with beacons indicated potential issue of excessive interaction with users which caused their negative reactions and rejection. Unexpected privacy intrusion by an advertiser can be seen as positive if it fits users behavior and provide added value either as benefits or entertainment [36].

In order to measure consumers' reaction to planned program and to do it as close to the act itself, we would plan exit interviews with shoppers - standardized questionnaire administered by the interviewer at the retail outlet once the shopper completes the shopping trip. Exit interviews would be conducted at the outlets activated with beacons. The sample would comprise of people triggered by the beaconthis would be the respondents selection or elimination criterion. Minimal realized sample in each outlet should be 30 respondents. Questionnaire duration would be up to 5 minutes. Base questions would aim to measure user satisfaction level, reasons for satisfaction or dissatisfaction, ease of use, relevance, expectations about similar activations in the future, perception of promoted brands (Coca-Cola and selected retailer as primary), together with standard demographics. Analysis would be done by standard descriptive statistics as well as variance analysis between satisfied and dissatisfied users.

\section{Measuring system functionality}

For the whole beacon activation program to work, following is required: user should have the smart phone on, SimplyTastly app should be installed, the phone has Bluetooth activated, beacon is active and communication algorithm is correct. The Coca-Cola system has another parallel project of testing beacons in coolers (for assets location and functionality tracking) in neighboring markets, which has a functional database and analytics and reporting interface. For the purpose of tracking the project in Serbia, new beacons would be added to the database tracking system which would enable monitoring of their performance.

Finally, project leader would have the responsibility to monitor cross functional implications of the project and run post activity review with all involved departments to assess the success and sustainability of implemented process and business practices to support the mass IoT implementation afterwards.

\section{v. Conclusion}

Technology development has given ground for IoT implementation in many areas, including marketing and retail. Possibility of communicating with the consumer or shopper in real time, at any stage of the purchase cycle and in relevant context, with personalized content and relevant benefit for the user are key reasons for IoT being seen as relevant and potent tool for marketing tactics. Additionally, IoT can improve business processes and user experience being applied for inventory management, retail logistics, payment systems, store employees management. Ultimately, it is expected that implementation of IoT delivers incremental added value and better business results vs standard ways of working.

The paper proposes a model of IoT application in marketing and retail that combines the business practice with technology solutions, connecting the manufacturers and retailers activating potential shoppers, combining the user interface and internal business processes. The paper also provides validation experiment of and activation project and its measurement system. The objective of the designed experiment is to validate the efficiency of IoT solution for driving sales conversion and as an additional connection point along the purchase cycle. The measurement system would give comprehensive insight into economic, psychological and technological effects. 
Model proposed in this paper is the initial step in bringing the theory and practice together to enable better understanding and ease of implementation for mass IoT solutions in marketing and retail industry. It is limited to this field, while there are many other business and non-business areas where IoT can bring benefits. Also, we have focused on specific measurement areas of economic, psychological and technological effects while a full ROI model for IoT solutions would deserve more research and validation, as it has significant financial and organizational requirements and implications for enterprises.

\section{References}

[1] B. Burton, D. A. Willis, Gartner's Hype Cycles for 2015: Five Megatrends Shift the Computing Landscape, https://www.gartner.com/doc/3111522?ref=SiteSearch\&refval=\&pcp =mpe, 12 August, 2015

[2] O. Vermesan, P. Friess, P. Guillemin, H. Sundmaeker, M. Eisenhauer, K. Moessner, F. Le Gall, P. Cousin, Internet of Things Strategic Research and Innovation Agenda, Internet of Things: Converging Technologies for Smart Environments and Integrated Ecosystems, 7151., 2013

[3] A. Al-Fuqaha, M. Guizani, M. Mohammadi, M. Aledhari, M. Ayyash, Internet of Things: A Survey on Enabling Technologies, Protocols, and Applications, IEEE Communication Surveys \& Tutorials, VOL. 17, NO. 4, Fourth Quarter 2015

[4] O. Vermesan, P. Friess, P. Guillemin, H. Sundmaeker, M. Eisenhauer, K. Moessner, M. Arndt, M. Spirito, P. Medagliani, R. Giaffreda, S. Gusmeroli, L. Ladid, M. Serrano, M. Hauswirth, G. Baldini, Internet of Things Strategic Research and Innovation Agenda, Internet of Things - From Research and Innovation to Market Deployment, 2014

[5] S. Chen, H. Xu, D. Liu, B. Hu, H. Wang, A Vision of IoT: Applications, Challenges, and Opportunities With China Perspective, IEEE Internet Of Things Journal, VOL. 1, NO. 4, August 2014

[6] V. Petrock, The Internet Of Things: Investment, Growth And Industry Outlook, eMarketer, May 2016

[7] T. Kambies, M. E. Raynor, D. M. Pankratz, G. Wadekar, Closing the digital divide: IoT in retail's transformative potential, Deloitte University Press, http://www2.deloitte.com/tr/en/pages/consumerbusiness/articles/internet-of-things-iot-retail-strategies.html, 14 January, 2016

[8] V. Petrock, The Complete Industry-by-Industry Guide for Marketers, eMarketer, May 2016

[9] V. Shankar, M. Kleijnen, S. Ramanathan, R. Rizley, S. Holland, S. Morrissey, Mobile Shopper Marketing: Key Issues, Current Insights, and Future Research Avenues, Journal of Interactive Marketing, 34 (2016) 37-48

[10] R. Pierdicca, D. Liciotti, M. Contigiani, E. Frontoni, A. Mancini, P. Zingaretti, Low Cost Embedded System For Increasing Retail Environment Intelligence, 2015 IEEE International Conference on Multimedia \& Expo Workshops (ICMEW, Date: June 29-July 32015

[11] J. Cai, Vertical Social Network for Retail Business Eco-system, A Proof of Concept, The Second International Conference on ETechnologies and Business on the Web (EBW2014) - Malaysia, March, 2014

[12] E. Frontoni, A. Mancini, P. Zingaretti, V. Placidi, Information Management for Intelligent Retail Environment: The Shelf Detector System, Information, 2014, 5, 255-271

[13] K. Nur, M. Morenza-Cinos, A. Carreras, R. Pous, Projection of RFID Obtained Product Information on a Retail Store's Indoor Panoramas, IEEE Intelligent Systems, 1541-1672/15

[14] N. L. Fantana, T. Riedel, J. Schlick, S. Ferber, J. Hupp, S. Miles, F. Michahelles, S. Svensson, IoT Applications - Value Creation for Industry, Internet of Things: Converging Technologies for Smart Environments and Integrated Ecosystems, 7-151., 2013

[15] C. Perera, R. Ranjan, L. Wang, S. U. Khan, A. Y. Zomaya, Big Data Privacy in the Internet of Things Era, IT Pro, Published by the IEEE Computer Society, May/June 2015, 1520-9202/15, 33-39

[16] L. Atzori, A. Iera, G. Morabito, From "Smart Objects" to "Social Objects": The Next Evolutionary Step of the Internet of Things, IEEE Communications Magazine, 0163-6804/14, January 2014, 97-105
[17] C. Perera, C. H. Liu, S. Jayawardena, M. Chen, A Survey on Internet of Things From Industrial Market Perspective, IEEE Access, Volume $2,2014,1660-1679$

[18] Y. Hsu, C. Lin, W. Chen, Design of a Sensing Service Architecture for Internet of Things with Semantic Sensor Selection, 2014 IEEE 11th International Conference on Ubiquitous Intelligence and Computing; 2014 IEEE 11th International Conference on Autonomic and Trusted Computing; 2014 IEEE 14th International Conference on Scalable Computing and Communications, 290 - 298

[19] N. M. Fong, Z. Fang, X. Luo, Geo-Conquesting: Competitive Locational Targeting of Mobile Promotions, Journal of Marketing Research, Vol. LII (October 2015), 726-735

[20] R. P. Minch, Location Privacy in the Era of the Internet of Things and Big Data Analytics, 2015 48th Hawaii International Conference on System Sciences, 1530-1605/15

[21] R. Gupta, R. Garg, Mobile Applications modelling and security handling in Cloud-centric Internet of Things, 2015 Second International Conference on Advances in Computing and Communication Engineering, 978-1-4799-1734-1/15, 2015 IEEE

[22] S.H. Choi, Y.X. Yang, B. Yang, H.H. Cheung, Item-level RFID for enhancement of customer shopping experience in apparel retail, Computers in Industry, 71 (2015) 10-23

[23] R. Gupta, R. Garg, Mobile Applications modelling and security handling in Cloud-centric Internet of Things, 2015 Second International Conference on Advances in Computing and Communication Engineering, 978-1-4799-1734-1/15, 2015 IEEE J. Gregory, The Internet of Things: Revolutionizing the Retail Industry, Accenture Strategy, 2015

[24] E. Pantano, H. Timmermans, What is smart for retailing?, Procedia Environmental Sciences; 12th International Conference on Design and Decision Support Systems in Architecture and Urban Planning, DDSS 2014, 22 ( 2014 ) $101-107$

[25] E. Kasznik, 5 ways the 'Internet of Things' transformed the vending machine, http://www.bizjournals.com/bizjournals/howto/technology/2015/04/the-internet-of-things-is-transformingvending.html?page=all, 10 April, 2015

[26] J. Liu, Y. Gu, S. Kamijo, Customer Behavior Recognition in Retail Store from Surveillance Camera, 2015 IEEE International Symposium on Multimedia, 978-1-5090-0379-2/15

[27] W. Zhou, F. Alexandre-Bailly, S. Piramuthu, Dynamic Organizational Learning with IoT and Retail Social Network Data, 2016 49th Hawaii International Conference on System Sciences, IEEE, 1530-1605/16

[28] D. R. Gnimpieba, A. Nait-Sidi-Moh, D. Durand, J. Fortina, Using Internet of Things technologies for a collaborative supply chain: Application to tracking of pallets and containers, Procedia Computer Science; International Workshop on Mobile Spatial Information Systems (MSIS 2015), 56 ( 2015 ) 550 - 557

[29] A ComQi White Paper, How the Internet of Things is Reinventing Retail, http://www.comqi.com/internet-things-reinventing-retail/, July 2015

[30] M. Radhakrishnan, A. Misra, R. K. Balan, Y. Lee, Smartphones \& BLE Services: Empirical Insights, 2015 IEEE 12th International Conference on Mobile Ad Hoc and Sensor Systems, 978-1-46739101-6/15

[31] J. Granjal, E. Monteiro, J. Sá Silva, Security for the Internet of Things: A Survey of Existing Protocols and Open Research Issues, IEEE Communication Surveys \& Tutorials, VOL. 17, NO. 3, Third Quarter 2015

[32] D. Kindström, C. Kowalkowski, E. Sandberg, Enabling service innovation: A dynamic capabilities approach, Journal of Business Research, (66), 8, 1063-1073, 2013

[33] C. Perera, A. Zaslavsky, Improve the Sustainability of Internet of Things Through Trading-based Value Creation, 2014 IEEE World Forum on Internet of Things (WF-IoT), 978-1-4799-3459-1/14

[34] Tata Consultancy Services, Internet of Things: The Complete Reimaginative Force, TCS Global Trend Study, July 2015

[35] J. A. Stankovic, Research Directions for the Internet of Things, IEEE Internet Of Things Journal, VOL. 1, NO. 1, February 2014

[36] R. Ström, M. Vendel, J. Bredican, Mobile marketing: A literature review on its value for consumers and retailers, Journal of Retailing and Consumer Services, Volume 21, Issue 6, November 2014, 10011012 